Thema des 27. September 2013 wird vormittags das Güterrecht sein, der Nachmittag wird dem Steuerrecht/Erbrecht/ Pflegerecht gewidmet. Die Kommission Zivil-, Familien- und Erbrecht wird das konkrete Programm anhand der genannten Rahmenbedingungen entwerfen. Ein weiterer Programmpunkt wird die Vorstellung der Neuauflage des Sonderheftes „65 Jahre djb“ sein. Ein Zimmerkontingent im Hotel Ibis Leipzig Zentrum (ca. 15 Min. zu Fuß vom BVerwG) zum Abruf durch die Teilnehmerinnen steht bereits zur Verfügung.

Der Bundesvorstand sammelte auch erste Ideen für eine Veranstaltung 2014 zum Gedenken an die Gründung des Deutschen Juristinnen-Vereins 1914 in Berlin. Der djb versteht sich als Nachfolgeorganisation dieses Vereins, der die Zulassung von Frauen zu juristischen Berufen erkämpft hatte, nach der Machtübernahme der Nazis aber seine Arbeit einstellen musste. Der erste Programmvorschlag stammt aus der K1: „Quotierung der Parlamente“ auf allen Ebenen, die Übertragung des Parité-Modells auf Deutschland.

\section{Verwaltung}

Ein Hauptbestandteil der Vorstandsarbeit sind Verwaltung und Finanzen. In der ersten Sitzung 2012 wurden der Wirtschaftsplan für das kommende Jahr und die Höhe der Zuweisungen an die Landesverbände und Regionalgruppen, letztere auf Antrag des RGB-Vorstands, beschlossen. Jahresabschluss, Mitgliederzahlen und Zuweisungshöhen der Landesverbände sind online. Jedes Jahr muss leider eine gewisse Zahl an Mitgliedern nach $\mathbb{S} 2$ der Satzung ausgeschlossen werden, da sie drei aufeinanderfolgende Jahresbeiträge nicht gezahlt haben. 2012 waren es sechs Mitglieder.

Zehn Jahre nach dem Umzug von Bonn nach Berlin stand im Januar 2012 die zwischenzeitlich abgeschlossene Renovierung der Geschäftsstellenräume an. Jetzt fehlt noch die „Ahnengalerie“: geplant sind Porträts der bisherigen 1. Vorsitzenden und Präsidentinnen.

\title{
2010-2012: Der djb mit Stand seit drei Jahren auf der WoMenPower
}

\section{Ute Wellner}

Vorstandsmitglied der RG Hannover; Juristin/Mediatorin, Langelsheim

Die WoMenPower 2012 hat sich wieder gelohnt! Im Rahmen der Hannover Messe findet seit Jahren immer am Freitag diese Veranstaltung statt. Diesmal war es der 27. April. Der djb, vertreten durch die Regionalgruppe Hannover, hatte dort zum nun dritten Mal nach 2010 und 2011 einen Stand. Der Fachkongress mit Ausstellenden aus verschiedensten frauenrelevanten Bereichen ist der größte in Deutschland. Mit Ständen vertreten waren Bundes- und Landesministerien, Frauen- und Gleichstellungsbeauftragte u.a. von Fachhochschulen und Universitäten sowie die Vernetzungsstelle Hannover, der Deut-

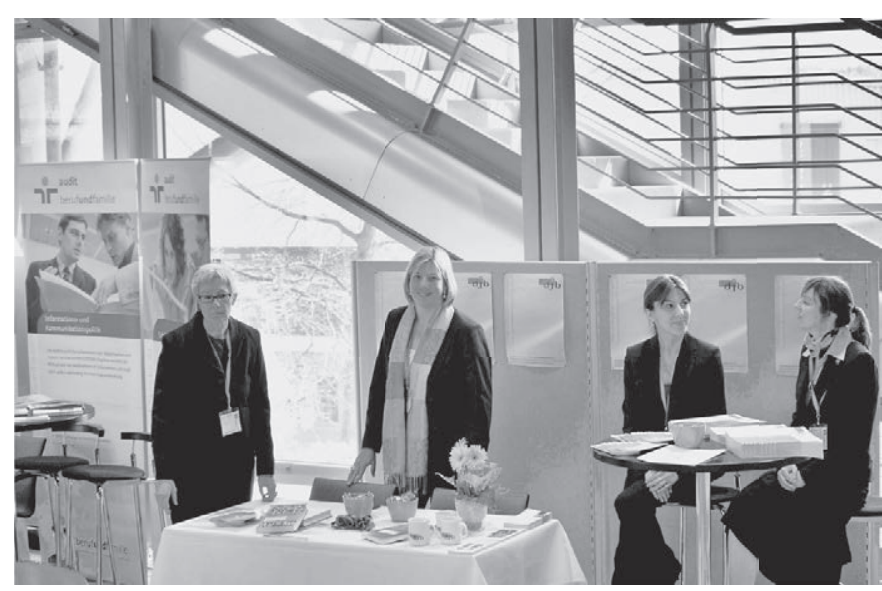

2010, v. I. n. r.: Ute Wellner, Birgit Kemming, Jelena Pap, Verena Schwach. sche Frauenrat zusammen mit einigen Landesfrauenräten, die Medienanstalten wie das ZDF, viele, viele Frauen mit ihren Unternehmungen, um nur einiges zu nennen. 1.200 Teilnehmende vorwiegend weiblichen Geschlechts waren diesmal gekommen, um sich zu informieren, auszutauschen und zu lernen oder kennenzulernen. Jedes Jahr steht die Veranstaltung unter einem Oberthema.

Das Thema in diesem Jahr war „Effizient Arbeiten und leben - design your future“. Die Teilnehmer/innen hatten sowohl am Vor- wie am Nachmittag die Möglichkeit, an Foren und Workshops teilzunehmen. Unter anderem machte Marion Knaths (Autorin von „Spiele mit der Macht“ und Inhaberin von sheBOSS, Hamburg) einen Workshop mit dem Titel: „Die zwei Seiten der gläsernen Decke“ - das Thema des djb-

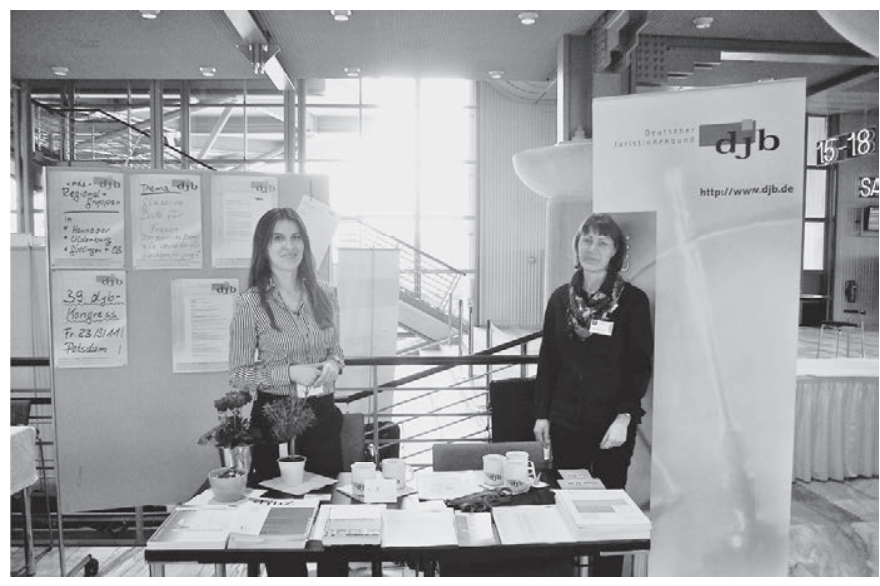

A 2011, v. I. n. r.: Jelena Pap, Yvonne Rokohl-Großmann. 


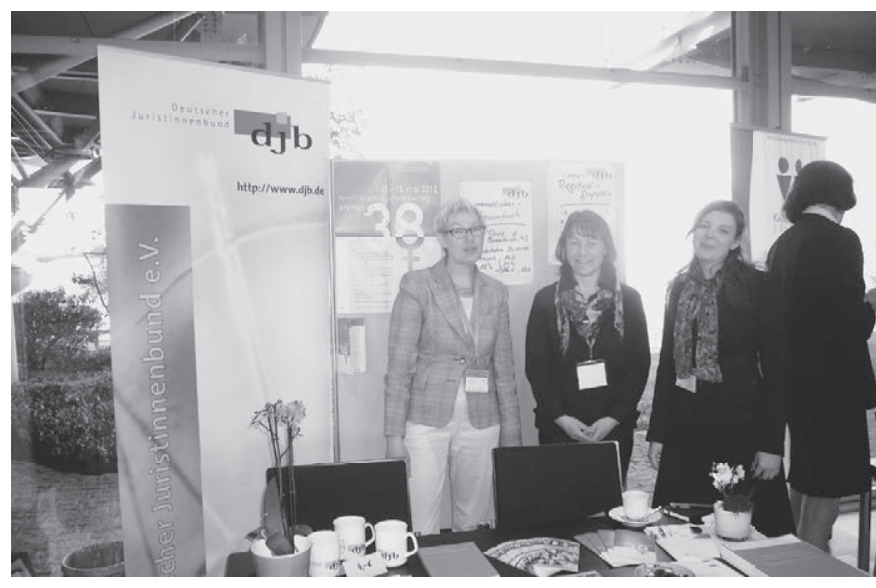

A 2012, v. I. n. r.: Ute Wellner, Yvonne Rokohl-Großmann, Verena Schwach.

Kongresses 2011. Workshop: Ausgebucht! Oder „Wie werde ich Aufsichtsrätin? Was macht eine Interim Managerin? Neue Karrierechancen 50 plus “ mit dem Deutschen Akademikerinnenbund und dessen AK Frauen in Naturwissenschaft \& Technik.

Unser Stand war wieder gut platziert. Zwischen „Landesfrauenrat Niedersachsen “ und ihrem Thema FrauenOrte auf der einen Seite und dem „Verband deutscher Unternehmerinnen e.V." auf der anderen. Wir, das waren Jelena Pap, Verena Schwach, Yvonne Rokohl-Großmann und Ute Wellner. Die Kolleginnen der anderen djb-Regionalgruppen des Landesverbands Niedersachsen mussten leider kurzfristig auf- grund beruflicher Termine absagen. Ansonsten wären Birgit Kemming wie 2010 und 2011 und Birgit Kersten auch dabei gewesen. Wir haben mit vielen interessierten Frauen gesprochen. Mit Rechtsanwältinnen, die in Großkanzleien arbeiten und Vernetzung suchen. Mit Müttern von Jurastudentinnen und Referendarinnen. Mit ehemaligen Hannoveranerinnen Erinnerungen und Erfahrungen über den jetzigen Standort ausgetauscht. Kontakte für Vernetzung und Zusammenarbeit wurden für Hannover geknüpft und Vereinbarungen für eine zukünftige Zusammenarbeit getroffen, unter anderem mit der Geschäftsführerin des Niedersächsischen Präventionsrates. Es kamen Frauen, aber auch mehrere Männer, die meinten, eine kostenlose, schnelle Rechtsberatung bekommen zu können oder ihren Frust über anwaltliche Beratung bei uns abzulassen. Ein Thema war diesmal beispielsweise die Patientenverfügung. Wir hatten einige djb-Exemplare dabei und hätten diese vielfach weitergeben können. Ein weiteres Thema war die Aktion „Frauen in die Aufsichtsräte“. Lob für die Aktion. Wir haben zwei Frauen kennengelernt, die sich beteiligt haben und uns ihre Erfahrungen mitgaben. Positive, aber es wurde auch Ärger bei den Standfrauen abgelassen. „Juristinnen in der DDR“ entwickelte sich zum Diskussionsthema am Stand.

Zum Ausklang der Veranstaltung gab es in schöner Tradition Brezeln und Sekt und das Statement vieler: wir sehen uns im nächsten Jahr wieder. Dann am Freitag, den 12. April 2013. Die drei Fotos zeigen den djb-Stand WoMenPower, Hannover in den Jahren 2010, 2011 und 2012.

\section{Juristinnen in der DDR}

\section{Eröffnung der Ausstellung in der Vertretung des Landes Sachsen-Anhalt bei der EU in Brüssel am 31. Mai 2012}

\section{Margarete Hofmann \\ djb-Vizepräsidentin, Brüssel}

Sehr geehrte Frau Ministerin Kolb, sehr geehrte Frau Professorin Will, sehr geehrte Frau Dr. Franz, liebe Kolleginnen und Freundinnen von der djb-Gruppe Brüssel, meine sehr geehrten Damen und Herren!

Der deutsche Juristinnenbund (djb) freut sich sehr, dass es gelungen ist, die Ausstellung „Juristinnen in der DDR“ nach Brüssel in die Landesvertretung Sachsen-Anhalt zu holen. Das ist insbesondere Ihrer Initiative und Aufgeschlossenheit, Frau Ministerin, und der Ihrer Mitarbeiterin Sabine Overkämping, die ich hier auch herzlich begrüßen möchte, zu verdanken. Mein Dank gilt auch Herrn André Lange von der Landesvertretung, der diese Veranstaltung bestens vorbereitet hat, sowie meinen Kolleginnen von der djb-Gruppe Brüssel, allen voran der Vorsitzenden, Dr. Annette Matthias, für die tatkräftige Unterstützung.

Frau Ministerin ist in ihren einleitenden Worten schon auf die aktuelle europaweite Debatte um Frauen in Führungspo- sitionen und den Streit um die Quote, der in Deutschland besonders erbittert ausgetragen wird, eingegangen. Was sie dazu in der Sache gesagt hat, kann der djb nur voll und ganz unterstreichen. Wir sind ohne Wenn und Aber für die Quote!

Ich selbst möchte in meinem Grußwort kurz erläutern, wie diese vom djb verantwortete Ausstellung, die als Wanderausstellung konzipiert ist, zustande gekommen ist. ${ }^{1}$ Ferner möchte ich auch auf Bedenken, die gegen diese Ausstellung geäußert wurden, eingehen.

Zunächst ist es sicherlich verständlich und nachvollziehbar, dass der djb als DER Verband der deutschen Juristinnen in Ost und West, mit nicht unbeträchtlichem Einfluss auf das rechts- und frauenpolitische Geschehen in Deutschland und auch in Europa, mehr als 20 Jahre nach der Wiedervereinigung sich dieses Themas angenommen hat. Dennoch war es

\footnotetext{
1 Die Schilderungen zum Werdegang und Inhalt der Ausstellung sind der Broschüre „Juristinnen in der DDR“: Rosemarie Will und Marion Röwekamp, hrsg. vom Deutschen Juristinnenbund, Berlin 2011 entnommen. Ausstellung und Broschüre wurden gefördert durch das Ministerium der Justiz des Landes Brandenburg.
} 